\title{
Classification of SD-OCT volumes with multi pyramids, LBP and HOG descriptors: application to DME detections
}

\author{
Khaled Alsaih*, Guillaume Lemaître*, Join Massich Vall*, Mojdeh Rastgoo*, Désiré Sidibé*, \\ Tien Y Wong ${ }^{\dagger}$, Ecosse Lamoureux ${ }^{\dagger}$, Dan Milea ${ }^{\dagger}$, Carol Y Cheung ${ }^{\dagger}$, \\ Fabrice Mériaudeau* ${ }^{*}$ \\ *LE2I UMR6306, CNRS, Arts et Métiers, Univ. Bourgogne Franche-Comté, \\ 12 rue de la Fonderie, 71200 Le Creusot, France \\ ${ }^{\dagger}$ Singapore Eye Research Institute, Singapore National Eye Center, Singapore \\ ${ }^{\ddagger}$ Centre for Intelligent Signal and Imaging Research (CISIR), Electrical \& Electronic Engineering Department, \\ Universiti Teknologi Petronas, 32610 Seri Iskandar, Perak, Malaysia \\ ฯCorresponding author: g.lemaitre58@gmail.com
}

\begin{abstract}
This paper deals with the automated detection of Diabetic Macular Edema (DME) on Optical Coherence Tomography (OCT) volumes. Our method considers a generic classification pipeline with preprocessing for noise removal and flattening of each B-Scan. Features such as Histogram of Oriented Gradients (HOG) and Local Binary Patterns (LBP) are extracted and combined to create a set of different feature vectors which are fed to a linear-Support Vector Machines (SVM) Classifier. Experimental results show a promising sensitivity/specificity of $\mathbf{0 . 7 5 / 0 . 8 7}$ on a challenging dataset.
\end{abstract}

\section{INTRODUCTION}

Eye diseases such as Diabetic Retinopathy (DR) and Diabetic Macular Edema (DME) are the most common causes of irreversible vision loss in individuals with diabetes. Just in United States alone, health care and associated costs related to eye diseases are estimated at almost $\$ 500 \mathrm{M}$ [1] with prevalent cases of DR expected to grow exponentially affecting over $300 \mathrm{M}$ people worldwide by 2025 [2]. Early detection and treatment of DR and DME play a major role to prevent adverse effects such as blindness. DME is characterized as an increase in retinal thickness within one disk diameter of the fovea center with or without hard exudates and sometimes associated with cysts [3]. Spectral Domain OCT (SD-OCT) [4] which images the depth of the retina with a high resolution and fast image acquisition is an adequate tool, compared to fundus images for DME identification [5, 6, 7]. As an example, Figure.11 shows one normal B-scan and one abnormal B-scans and clearly illustrate the fact that lesions such as cysts can easily be spotted via Optical Coherence Tomography (OCT) imaging. Automated diagnosis on OCT imaging is rather new and most of the pioneer works on OCT image analysis have focused on the problem of retinal layers segmentation, which is a necessary step for retinal thickness measurements $[8,9,10]$ and has also recently proven to be quite efficient for cysts segmentation [11]. However, very few works, up to our knowledge, have addressed the specific problem of DME and its associated features detection from OCT images. In this paper we propose a solution for automated detection of

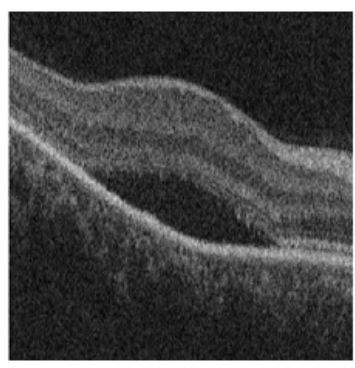

(a) DME-case

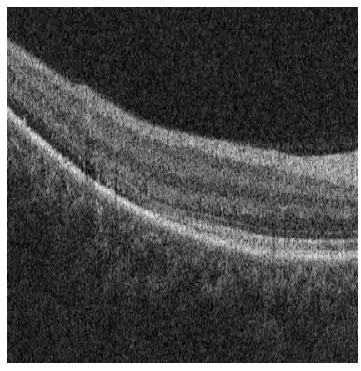

(b) normal-case
Fig. 1. DME and normal case of OCT B-scans.

DME on OCT volumes.

Following this introduction, the rest of the paper is organized as follows, Sect.II] will present some related works, Sect. III is related to the data collection, Sect [IV and Sect. $\mathrm{V}$ are respectively dedicated to our methodology and the obtained results. The paper ends with a short discussion and some conclusion in Sect.VI.

\section{RELATED WORK}

Srinivasan et al. proposed a classification method to distinguish normal OCT volumes from DME and Age-related Macular Degeneration (AMD) volumes [12]. The OCT images are pre-processed by reducing the speckle noise by enhancing the sparsity in a transform-domain and flattening the retinal curvature to reduce the inter-patient variations. Then, Histogram of Oriented Gradients (HOG) are extracted for each slice of a volume and fed to a linear Support Vector Machines (SVM). This method was applied onto a dataset of 45 patients equally subdivided into the three aforementioned classes and led to a correct classification rate of $100 \%, 100 \%$ and $86.67 \%$ for normal, DME and AMD patients, respectively. The images that were used in their paper, are publically available but are already preprocessed (noise removed), do not offer a huge variability in term of DME lesions, have different sizes for the OCT volumes, and some of them (without specifying which) have been excluded 


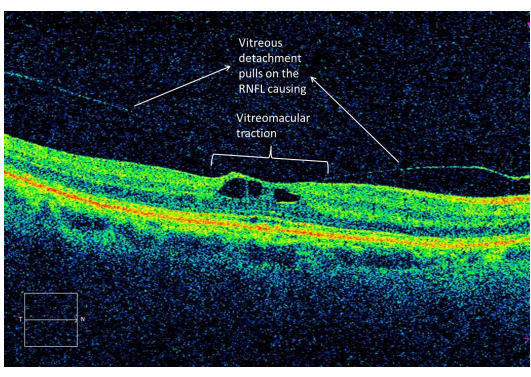

(a) Vitreomacular traction.

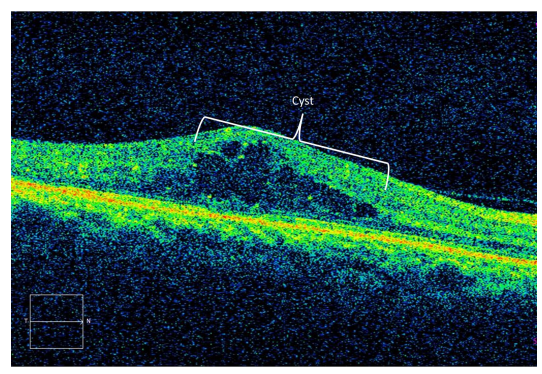

(d) Cyst spaces and hard exudates, causing central retinal thickening.

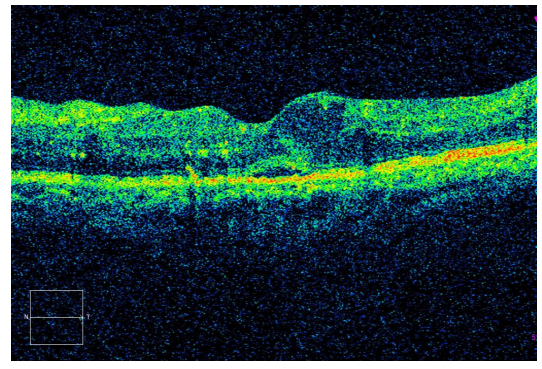

(g) Cyst spaces, causing retinal thickening.

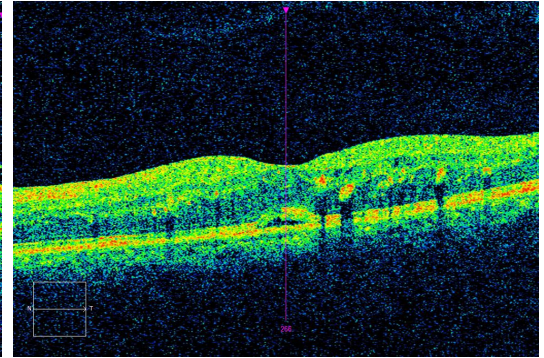

(b) Rethinal thickening.

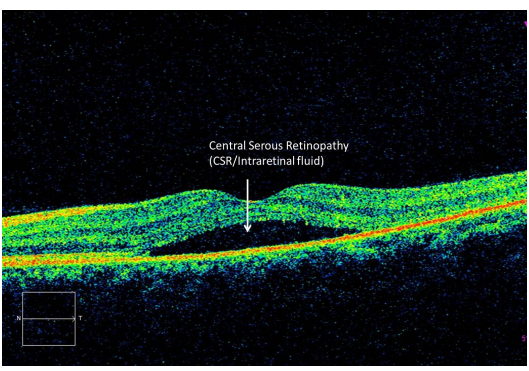

(e) CSR (subretinal fluid), causing central and parafoveal thickening.

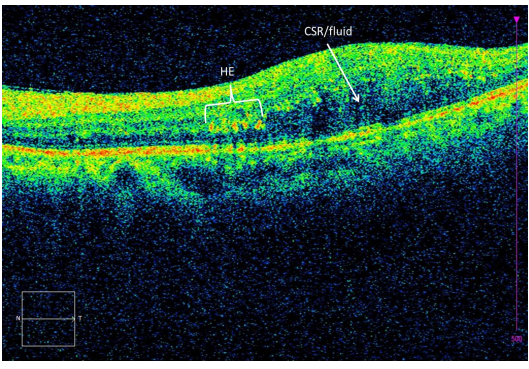

(h) CSR and hard exudates, causing retinal thickening.

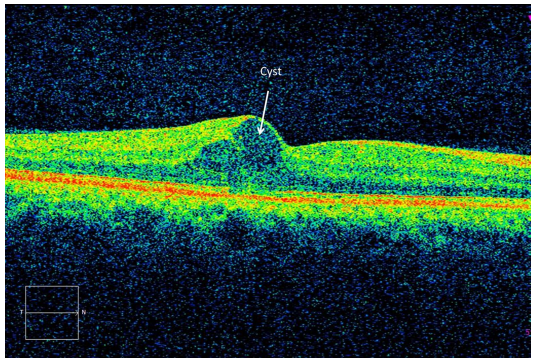

(c) Cyst spaces, causing central and parafoveal retina thickening.

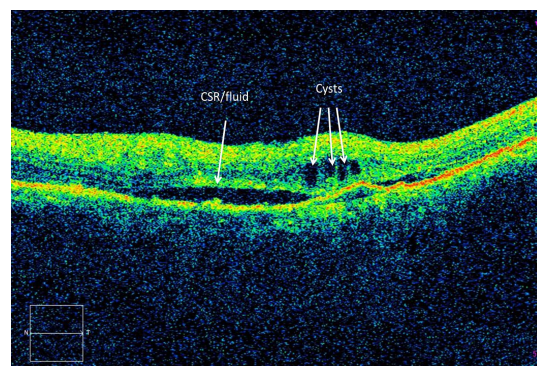

(f) CSR, hard exudates and cyst spaces.

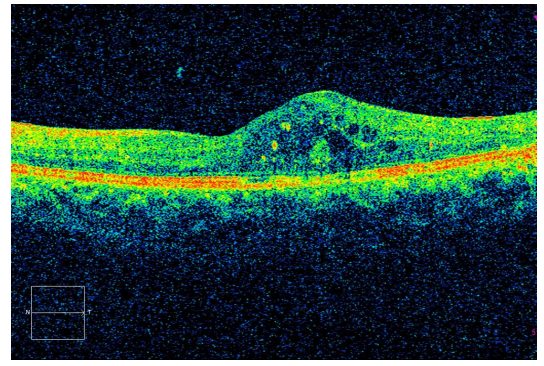

(i) Cyst spaces causing parafoveal thickening

Fig. 2. Examples of DME cases in Singapore Eye Research Institute (SERI) dataset.

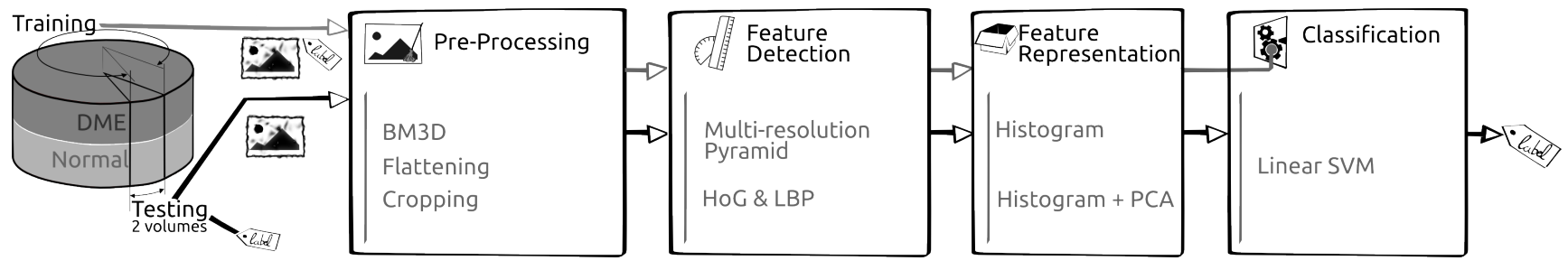

Fig. 3. Proposed classification pipeline.

for the training phase; all these reasons prevent us from using this dataset to benchmark our work. Venhuizen et al. recently proposed a method for OCT images classification using the Bag-of-Words (BoW) models [13]. The method starts with the detection and selection of keypoints in each individual B-scan, by keeping the most salient points corresponding to the top $3 \%$ of the vertical gradient values. Then, a $9 \times 9$ pixels texton is extracted around each keypoint, and Principal Component Analysis (PCA) is applied to reduce the dimension of every texton from 81 to 9 . All extracted feature vectors are used to create a dictionnary using kmeans clustering. Then, each OCT volume is represented as an histogram that captures the codeword occurrences. These histograms are used as feature vector to train a Random Forest (RF) classifier with a maximum of 100 trees. The method was used to classify OCT volumes between AMD and normal cases and achieved an Area Under the Curve (AUC) of 0.984 with a dataset of 384 OCT volumes [14]. Liu et al. proposed a methodology for detecting macular pathology in OCT images using Local Binary Patterns (LBP) 
and gradient information as attributes [15]. The method starts by aligning and flattening the images and creating a 3-level multi-scale spatial pyramid. The edge and LBP histograms are then extracted from each 80 block of every level of the pyramid. All the obtained histograms are concatenated into a global descriptor whose dimensions are reduced using PCA. Finally a SVM with an Radial Basis Function (RBF) kernel is used as classifier. The method achieved good results in detection OCT scan containing different pathology such as DME or AMD, with an AUC of 0.93 using a dataset of 326 OCT scans. Lemaître et al. developed a classification framework based on LBP features to describe the texture of OCT images and dictionary learning using the BoW models [16]. They proposed to extract 2D and 3D LBP features from OCT images and volumes, respectively. The LBP descriptors are either extracted from the entire sample or local patches within individual samples. Numerous experiments were conducted and the authors achieved a sensitivity and specificity of $81.2 \%$ and $93.2 \%$ for their best configuration. On the same dataset, a different approach which consists in addressing this issue as an anomaly detection problem was recently proposed by Sankar et al. [17]. In their method, the authors propose a technique that not only allow the classification of the OCT volume, but also enables the identification of the individual diseased B-scans inside the volume. This approach is based on modeling the appearance of normal OCT images with a Gaussian Mixture Model (GMM) and detecting abnormal OCT images as outliers. The classification of an OCT volume is based on the number of detected outliers. Experimental results with two different datasets show that the proposed method achieves a sensitivity and a specificity of $80 \%$ and $93 \%$ on the first dataset, and $100 \%$ and $80 \%$ on the second one. The proposed method achieves better classification performance than other recently published work but it requires to tune the GMM parameters and it should be tested on a larger database.

\section{DATA}

The datasets used in this study were acquired by the SERI, using CIRRUS TM (Carl Zeiss Meditec, Inc., Dublin, CA) SD-OCT device [4]. The datasets consist of 32 OCT volumes (16 DME and 16 normal cases). Each volume contains 128 B-scan with resolution of $512 \times 1024$ px. All SDOCT volumes are read and assessed by trained graders and identified as normal or DME cases based on evaluation of retinal thickening, hard exudates, intraretinal cystoid space formation and subretinal fluid as presented in the table below. Within the DME dataset, a large number of lesions were selected to create a rather complete DME dataset. The volumes and sample numbers for each volume are presented below (Fig.2):

\section{Methodology}

Our approach is based on the original paper of Srinivasan et al. [12] to which a feature extraction procedure (PCA) is added, as proposed by Venhuizen et al. [14]. Our general pipeline is presented on Fig. 3

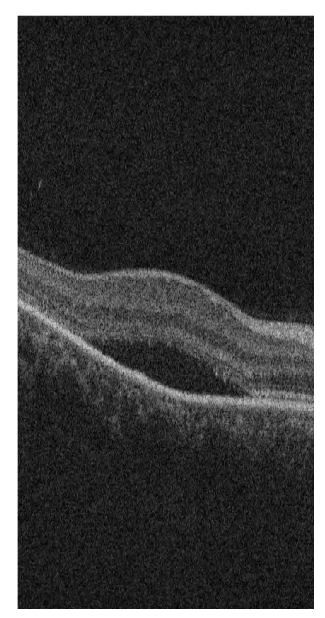

(a) Original

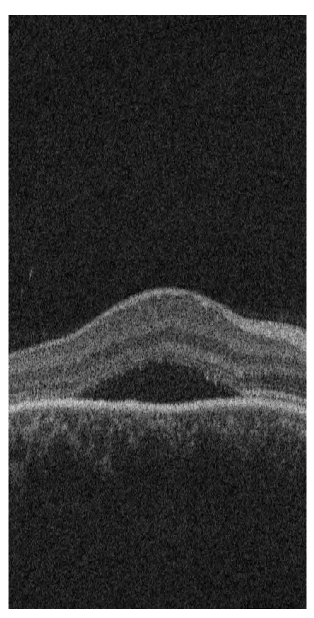

(b) Flatten
Fig. 4. Original and flatten image.

First the image are preprocessed to remove the noise with the BM3D algorithm developed by Dabov et al. [18]; they are then flattened and cropped as described by Srinivisan et al. [12] (see Fig.4). On the cropped image, we extract HOG features [19] as well as LBP features either in their standard version [20] or their rotation invariant version [21] with different neighborhoods. Furthermore, to consider structures at multiple scale levels, both HOG and LBP features vector are extracted at four levels of the multiscale Gaussian lowpass image pyramid. For each feature, HOG or LBP the histogram are concatenated into a single feature vector for each image. Then the obtained histograms are either fed directly to a linear SVM classifier or reduced with PCA. We attempt to combine HOG and LBP feature vectors or use them separately as explain in the next section.

\section{EXPERIMENTAL RESULTS}

The validation is done on a leave two patient out basis (one DME volume, one normal volume). As 16 volumes were provided, the experiments were performed 16 times and the results averaged and presented in the following table. $R I$ stands for Rotation Invariant LBP, whereas $N R I$ stands for Non Rotation Invariant (i.e. $\mathrm{LBP}_{8-N R I}$ stands for 8 neighbors Non Rotation Invariant). The feature vectors were reduced to the size of 40 after PCA (i.e. $\mathrm{HOG}_{P C A}$ ) was applied onto the original HOG feature vector and 20 for each of the LBP extracted feature vector. Different experiments were carried out and their results are summarized below (see Table.I).

\section{Discussion AND CONCLUSION}

We presented an automatic classification framework for SD-OCT volumes in order to indentify DME volumes versus normal Volumes. In this regard, we investigated a generic pipeline including preprocessing, feature extraction and feature reduction and feature combination. The best results were obtained for HOG feature vectors. We plan to extend our work with a BoW approach as well as we intend to study 
TABLE I

OUTLINE AND SUMMARY OF THE PERFORMED EXPERIMENTS AND OBTAINED RESULTS.

\begin{tabular}{lccccc}
\hline Features & \multicolumn{5}{c}{ Evaluations } \\
\cline { 2 - 6 } & Sensitivity & Specificity & Precision & F1-score & Accuracy \\
\hline $\mathrm{HOG}$ & 0.69 & 0.94 & 0.91 & 0.81 & 0.78 \\
$\mathrm{HOG}^{P C A}$ & 0.75 & 0.87 & 0.85 & 0.80 & 0.81 \\
$\mathrm{HOG}^{P C A}+\mathrm{LBP}_{8-N R I}^{P C A}$ & 0.69 & 0.75 & 0.73 & 0.71 & 0.72 \\
$\mathrm{HOG}^{P C A}+\mathrm{LBP}_{8-N R I}^{P C A}+\mathrm{LBP}_{16-N R I}^{P C A}+\mathrm{LBP}_{24-N R I}^{P C A}$ & 0.62 & 0.75 & 0.71 & 0.66 & 0.68 \\
$\mathrm{HOG}^{P C A}+\mathrm{LBP}_{8-R I}^{P C A}+\mathrm{LBP}_{16-R I}^{P C A}+\mathrm{LBP}_{24-R I}^{P C A}$ & 0.69 & 0.81 & 0.78 & 0.73 & 0.75 \\
\hline
\end{tabular}

other classifiers (RBF-SVM, RF) in a near future. Also, we are currently working on the extension of the dataset so as to make it more challengeable and open to the community.

\section{ACKNOWLEDGMENT}

The authors would like to acknowledge the financial support of the PHC Merlion from France and Singapore. The authors would like to acknowledge the Regional Burgundy Council which partially financially supported the current project under the PARI scheme 2.

\section{REFERENCES}

[1] S. Sharma, A. Oliver-Hernandez, W. Liu, and J. Walt, "The impact of diabetic retinopathy on health-related quality of life," Current Opinion in Ophtalmology, vol. 16, pp. 155-159, 2005.

[2] S. Wild, G. Roglic, A. Green, R. Sicree, and H. King, "Global prevalence of diabetes estimates for the year 2000 and projections for 2030," Diabetes care, vol. 27, no. 5, pp. 1047-1053, 2004.

[3] Early Treatment Diabetic Retinopathy Study Group, "Photocoagulation for diabetic macular edema: early treatment diabetic retinopathy study report no 1," JAMA Ophthalmology, vol. 103, no. 12, pp. 1796$1806,1985$.

[4] T. C. Chen, B. Cense, M. C. Pierce, N. Nassif, B. H. Park, S. H. Yun, B. R. White, B. E. Bouma, G. J. Tearney, and J. F. de Boer, "Spectral domain optical coherence tomography: ultra-high speed, ultra-high resolution ophtalmic imaging," JAMA Ophthalmology, vol. 123, no. 12, pp. 1715-1720, 2005.

[5] M. D. Abràmoff, M. K. Garvin, and M. Sonka, "Retinal imaging and image analysis," Biomedical Engineering, IEEE Reviews in, vol. 3, pp. 169-208, 2010.

[6] E. Trucco, A. Ruggeri, T. Karnowski, L. Giancardo, E. Chaum, J. P. Hubschman, B. al Diri, C. Y. Cheung, D. Wong, M. Abramoff et al., "Validating retinal fundus image analysis algorithms: Issues and a proposalvalidating retinal fundus image analysis algorithms," Investigative ophthalmology \& visual science, vol. 54, no. 5, pp. 35463559, 2013.

[7] L. Giancardo, F. Meriaudeau, T. P. Karnowski, K. W. Tobin Jr, E. Grisan, P. Favaro, A. Ruggeri, and E. Chaum, "Textureless macula swelling detection with multiple retinal fundus images," Biomedical Engineering, IEEE Transactions on, vol. 58, no. 3, pp. 795-799, 2011.

[8] S. J. Chiu, X. T. Li, P. Nicholas, C. A. Toth, J. A. Izatt, and S. Farsiu, "Automatic segmentation of seven retinal layers in sdoct images congruent with expert manual segmentation," Optics express, vol. 18, no. 18, pp. 19413-19428, 2010.

[9] M. K. Garvin, M. D. Abràmoff, X. Wu, S. R. Russell, T. L. Burns, and M. Sonka, "Automated 3-d intraretinal layer segmentation of macular spectral-domain optical coherence tomography images," Medical Imaging, IEEE Transactions on, vol. 28, no. 9, pp. 1436-1447, 2009.
[10] M. K. Garvin, M. D. Abràmoff, R. Kardon, S. R. Russell, X. Wu, and M. Sonka, "Intraretinal layer segmentation of macular optical coherence tomography images using optimal 3-d graph search," IEEE Transactions on Medical Imaging, vol. 27, no. 10, pp. 1495-1505, 2008.

[11] I. Oguz, L. Zhang, M. D. Abràmoff, and M. Sonka, "Optimal retinal cyst segmentation from oct images," in SPIE Medical Imaging. International Society for Optics and Photonics, 2016, pp. 97 841E$97841 \mathrm{E}$.

[12] P. P. Srinivasan, L. A. Kim, P. S. Mettu, S. W. Cousins, G. M. Comer, J. A. Izatt, and S. Farsiu, "Fully automated detection of diabetic macular edema and dry age-related macular degeneration from optical coherence tomography images," Biomedical optics express, vol. 5, no. 10, pp. 3568-3577, 2014.

[13] J. Sivic and A. Zisserman, "Video google: a text retrieva approach to object matching in videos," in IEEE ICCV, 2003, pp. 1470-1477.

[14] F. G. Venhuizen, B. van Ginneken, B. Bloemen, M. J. van Grinsven, R. Philipsen, C. Hoyng, T. Theelen, and C. I. Sánchez, "Automated age-related macular degeneration classification in oct using unsupervised feature learning," in SPIE Medical Imaging. International Society for Optics and Photonics, 2015, pp. 94 141I-94 141I.

[15] Y.-Y. Liu, M. Chen, H. Ishikawa, G. Wollstein, J. S. Schuman, and J. M. Rehg, "Automated macular pathology diagnosis in retinal oct images using multi-scale spatial pyramid and local binary patterns in texture and shape encoding," Medical image analysis, vol. 15, no. 5, pp. 748-759, 2011.

[16] G. Lemaître, M. Rastgoo, J. Massich, S. Sankar, F. Mériaudeau, and D. Sidibé, "Classification of sd-oct volumes with lbp: Application to dme detection," in Proceedings of the Ophthalmic Medical Image Analysis Second International Workshop, OMIA 2015, Held in Conjunction with MICCAI 2015, 2015.

[17] S. Sankar, D. Sidibé, Y. Cheung, T. Wong, E. Lamoureux, D. Milea, and F. Meriaudeau, "Classification of sd-oct volumes for dme detection: an anomaly detection approach," in SPIE Medical Imaging. International Society for Optics and Photonics, 2016, pp. $978520-$ 978520.

[18] K. Dabov, A. Foi, V. Katkovnik, and K. Egiazarian, "Image denoising by sparse 3-d transform-domain collaborative filtering," Image Processing, IEEE Transactions on, vol. 16, no. 8, pp. 2080-2095, 2007.

[19] N. Dalal and B. Triggs, "Histograms of oriented gradients for human detection," in Computer Vision and Pattern Recognition, 2005. CVPR 2005. IEEE Computer Society Conference on, vol. 1. IEEE, 2005, pp. 886-893.

[20] T. Ojala, M. Pietikäinen, and T. Mäenpää, "Multiresolution gray-scale and rotation invariant texture classification with local binary patterns," Pattern Analysis and Machine Intelligence, IEEE Transactions on, vol. 24, no. 7, pp. 971-987, 2002.

[21] G. Zhao, T. Ahonen, J. Matas, and M. Pietikäinen, "Rotation-invariant image and video description with local binary pattern features," Image Processing, IEEE Transactions on, vol. 21, no. 4, pp. 1465-1477, 2012. 